\title{
C-reactive protein as a marker of ventilator-associated pneumonia resolution: a pilot study
}

\author{
P. Póvoa, L. Coelho, E. Almeida, A. Fernandes, R. Mealha, P. Moreira and H. Sabino
}

ABSTRACT: The aim of this study was to evaluate C-reactive protein (CRP) levels, body temperature and white cell count (WCC) after prescription of antibiotics in order to describe the clinical resolution of ventilator-associated pneumonia (VAP).

A cohort of 47 VAP patients with microbiological confirmation of disease was assessed. CRP levels, body temperature and WCC were monitored daily.

On day 4 of the antibiotic therapy, the CRP level of survivors was 0.62 times the initial value, whereas, in nonsurvivors, it was 0.98 . Body temperature and WCC remained almost unchanged. By day 4, a CRP of $>0.6$ times the initial level was a marker of poor outcome (sensitivity 0.92 ; specificity 0.59). Patients were divided according to their CRP patterns of response to antibiotics: fast response, slow response, nonresponse, and biphasic response. All patients with fast and slow response patterns survived, whereas those showing nonresponse and a biphasic response pattern exhibited a mortality of 78 and $75 \%$, respectively. The adequacy of the initial antibiotic therapy had a marked influence on the rate of CRP decrease, as well as on mortality.

In conclusion, daily C-reactive protein measurements after antibiotic prescription were useful in the identification, as early as day 4 , of ventilator-associated pneumonia patients with poor outcome. The identification of the pattern of C-reactive protein response to antibiotics was useful in the recognition of individual clinical course, improving or worsening, as well as of the rate of improvement.

KEYWORDS: C-reactive protein, intensive care unit, outcome, temperature, ventilator-associated pneumonia, white cell count

V entilator-associated pneumonia (VAP) is the most frequent intensive care unit (ICU)-acquired infection among patients undergoing mechanical ventilation [1]. As a result, VAP prolongs the length of ICU stay and, in addition, is associated with a $20-30 \%$ increase in the risk of death [2-4]. Therefore, mechanically ventilated patients are aggressively monitored in order to diagnose VAP prematurely. Currently, the diagnosis of VAP is based on a combination of clinical, radiological and bacteriological criteria [5]. These criteria are very sensitive but poorly specific and, therefore, antibiotics are often inappropriately prescribed [4].

The evaluation of VAP response to antibiotic therapy relies on the resolution of the same criteria used in the diagnosis [4-6]. Bacterial eradication has been proposed as a criterion of improvement [7]; however, these results were recently challenged [5]. Chest radiography is also of limited value as initial deterioration is common $[6,8]$. Clinical parameters, such as body temperature, blood pressure, white cell count (WCC), respiratory frequency, arterial oxygen tension $\left(\mathrm{Pa}_{2} \mathrm{O}_{2}\right) /$ inspiratory oxygen fraction $\left(\mathrm{FI}, \mathrm{O}_{2}\right)$ ratio and tracheobronchial secretions, have also been used to assess response to therapy [4-6, 8, 9]. Recently, a combination of these parameters, the clinical pulmonary infection score (CPIS), was tested in the evaluation of VAP response to therapy [8].

C-reactive protein (CRP) belongs to a family of proteins named pentraxins. These proteins are stably conserved throughout vertebrate evolution, suggesting a central role in immunological response [10]. Several studies have shown that CRP is useful in the diagnosis of sepsis in different clinical situations [11], although data are sparse regarding its behaviour in septic patients after the institution of antibiotics [1214]. Four different patterns of CRP progression in

\section{AFFILIATIONS}

Intensive Care Unit, Garcia de Orta Hospital, Almada, Portugal.

CORRESPONDENCE

P. Póvoa

Unidade de Cuidados Intensivos

Hospital Garcia de Orta

Av. Prof. Torrado da Silva

2800-525 Almada

Portugal

Fax: 351212957004

E-mail: povoap@netcabo.pt

Received:

June 152004

Accepted after revision:

December 132004 
response to antibiotics, as well as relationships with prognosis, have already been described $[12,13]$.

The aim of the present study was to assess the value of serial CRP determinations in comparison to commonly used infectious markers, such as body temperature and WCC, after prescription of antibiotics in the evaluation of resolution of $\mathrm{VAP}$, in order to recognise, early in the clinical course, patients with good and bad outcome, as well as to identify the individual pattern of CRP response to antibiotics. Since the adequacy of antibiotic therapy is a risk factor for hospital mortality $[15,16]$, the effect of the appropriateness of antibiotic therapy on CRP changes over time, as well as on outcome, was also assessed.

\section{METHODS AND MATERIALS Study subjects}

A prospective observational cohort study was conducted between November 2001 and December 2002. The ICU of Garcia de Orta Hospital (Almada, Portugal) is an eight-bed ward, harbouring a mixed population of medical, surgical and trauma patients. All patients who were aged $\geqslant 18$ yrs and received mechanical ventilation for $>72 \mathrm{~h}$ were enrolled. The Ethics Committee of Garcia de Orta Hospital approved the study design.

\section{Study design}

The data collected included admission diagnosis, past medical history and vital signs. CRP concentration, body temperature, WCC and Sequential Organ Failure Assessment (SOFA) score [17] were recorded every day. Patients were evaluated daily for evidence of VAP. In the presence of a clinical diagnosis of VAP, samples were collected for bacteriological culture, namely bronchoalveolar lavage (BAL) or protected specimen brush and blood cultures. After clinical VAP diagnosis, all patients received empirical antibiotic therapy. Patients with a clinical diagnosis of VAP but without microbiological documentation were not included in the final analysis.

Blood samples were obtained via an arterial line on admission and, subsequently, every morning at 07:00 h. Measurement of CRP was performed by means of an immunoturbidimetric method using a commercially available kit (Tina-quant CRP; Roche Diagnostics, Mannheim, Germany). The precision of the assay measured by means of the intra- and interassay coefficient of variation was $<7 \%$, the sensitivity $0.1 \mathrm{mg} \cdot \mathrm{dL}^{-1}$ and the detection limit $0.3 \mathrm{mg} \cdot \mathrm{dL}^{-1}$.

For the purposes of time-dependent analysis, day 0 (D0) was defined as the day of VAP clinical diagnosis, as well as when the empirical antibiotic therapy for VAP was started. The following days were successively defined as D1, D2, etc.

Withdrawal of the inflammatory stimulus results in a sharp decrease in serum CRP concentration, similar to first-order elimination kinetics [10]. As a result, time-dependent analysis of the relative CRP concentration (CRP ratio) was also performed. The CRP ratio was calculated in relation to D0 CRP concentration.

Patients were followed-up until pneumonia was cured and they were sufficiently clinically stable to be discharged from the ICU or until death. The progression of CRP concentration,
CRP ratio, body temperature and WCC throughout the course of VAP was analysed, comparing survivors and nonsurvivors.

\section{Definitions}

VAP was defined as a new and persistent radiographic infiltrate plus at least two of the following criteria: 1) body temperature of $>38$ or $<36^{\circ} \mathrm{C}$; 2) WCC of $>10 \times 10^{3}$ or $<4 \times 10^{3}$ cells $\cdot \mu \mathrm{L}^{-1}$; and 3) purulent tracheal aspirate. In addition, it was necessary to have microbiological documentation, with the growth of $\geqslant 1 \times 10^{4}$ colony-forming units $(\mathrm{cfu}) \cdot \mathrm{mL}^{-1}$ of a microorganism on BAL or $\geqslant 1 \times 10^{3} \mathrm{cfu} \cdot \mathrm{mL}^{-1}$ on protected specimen brush and/or the isolation of a potential pathogen from blood cultures $[4,18,19]$.

Adequate antibiotic therapy was defined as when, in the empirical therapy prescribed by the onset of VAP, at least one antibiotic covers all of the pathogens isolated, as determined by the sensitivity pattern in the antibiogram. In patients started with initially inadequate treatment, antibiotics were changed according to the pathogen isolated and antimicrobial susceptibility testing.

CRP patterns of response to antibiotic therapy after prescription of antimicrobials were modifications of those previously published: a fast response occurred when the CRP ratio at D4 was $<0.4$ relative to D0 CRP; a slow response was characterised by a continuous and slow decrease in CRP ratio; nonresponse was when the CRP ratio remained at $\geqslant 0.8$; and a biphasic response was characterised by an initial CRP ratio decrease to levels of $<0.8$, followed by a secondary rise to values of $\geqslant 0.8$ (fig. 1) $[12,13]$. VAP patients were retrospectively divided into four groups according to their pattern of CRP response.

\section{Analysis}

Continuous variables are presented as mean \pm SD, unless otherwise stated. Comparisons between groups were performed using the parametric unpaired and paired t-test, or the nonparametric Mann-Whitney U-test and Wilcoxon signedrank test for continuous variables according to data distribution. The Shapiro-Wilk test was used for normality assessment. The Chi-squared test was used to carry out comparisons between categorical variables. Time-dependent analysis of different variables was performed via General Linear Model univariate repeated-measures analysis using a split-plot design approach. Differences at different time points were determined by calculation of contrast coefficients.

Receiver-operating characteristic (ROC) curves were plotted for CRP ratio, body temperature and WCC on D4 of antimicrobial therapy. The indicative accuracy of these variables at D4 was assessed by calculation of the area under the curve (AUC), as described elsewhere [20]. In medical practice, a diagnostic test with an AUC of $<0.75$ is regarded as noncontributive [21]. Comparison of the AUC of two variables was performed using the method of HANLEY and MCNEIL [22]. Regarding nonsurvivors, D4 data refer only to patients still alive on that day.

In order to identify the variations in infectious markers, specifically CRP ratio, body temperature and WCC at D4 (independent variables), best predicting VAP outcome (dependent variable) during antibiotic therapy, a multivariable 

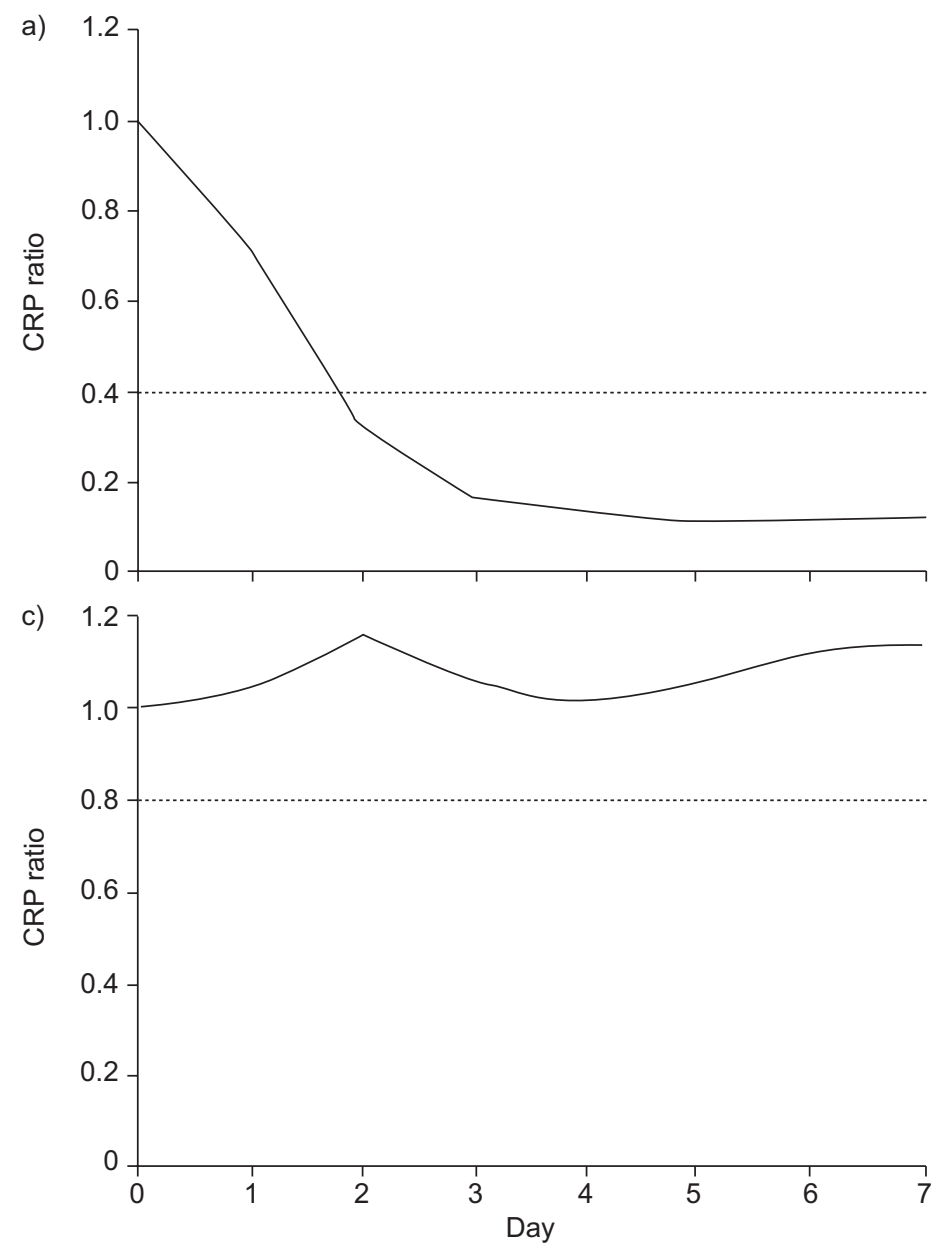

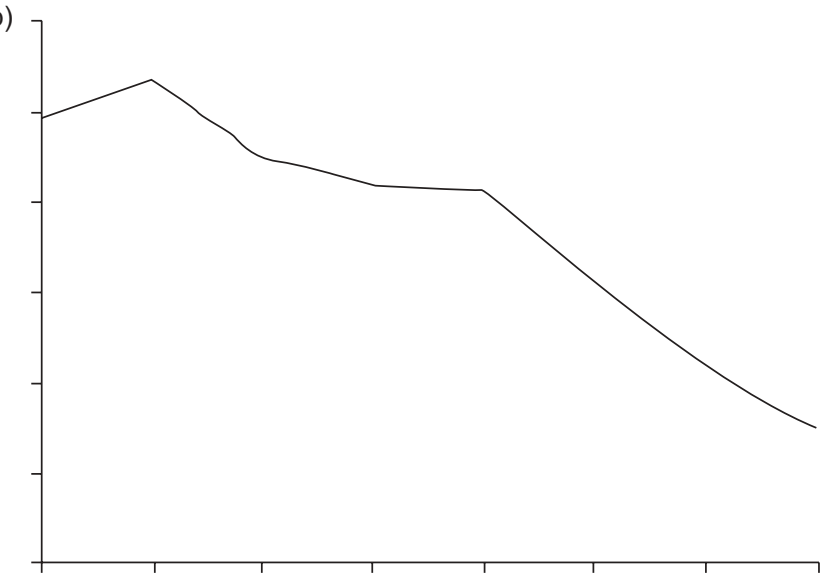

d)

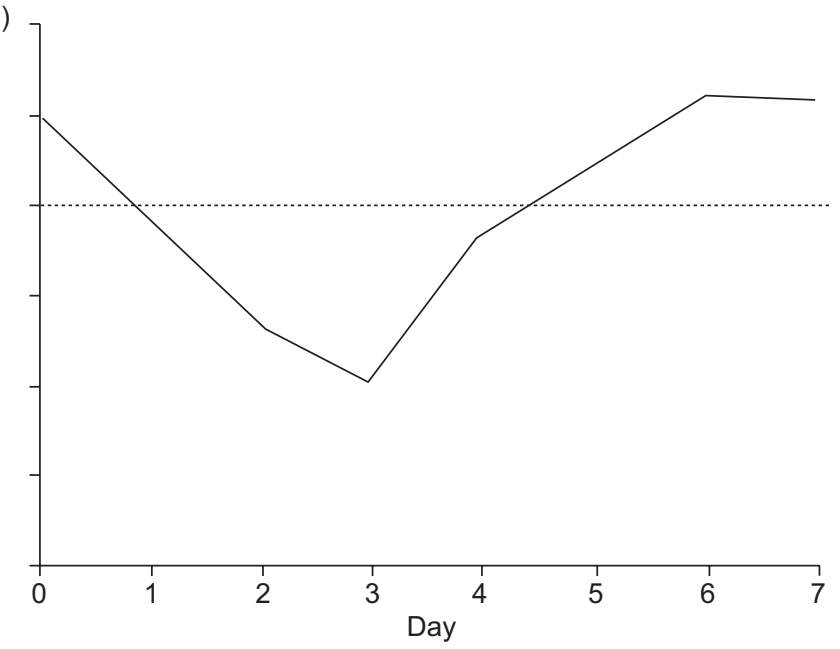

FIGURE 1. C-reactive protein (CRP) ratio patterns of response of four different ventilator-associated pneumonia patients after prescription of antimicrobials: a) fast response (CRP ratio at day $4<0.4$ ); b) slow response (continuous and slow decrease in CRP ratio); c) nonresponse (CRP ratio remains $\geqslant 0.8$ ); and d) biphasic response (CRP ratio decreases to $<0.8$, followed by a secondary rise to $\geqslant 0.8$ ). CRP ratio: CRP concentration relative to day 0 CRP concentration. $\cdots \cdots$ : CRP cut-off value for response type.

logistic regression model was constructed. In order to control for potential confounding factors, age, sex, Acute Physiology, Age, and Chronic Health Evaluation (APACHE) II score, and D0 SOFA score were included in the initial model. Backward stepwise variable elimination was then performed, with a p-value of $<0.05$ as a requirement for acceptance, in order to develop the final model. Model calibration and discrimination were assessed using the Hosmer-Lemeshow goodness-of-fit test and the c-statistic, respectively. Results are reported as adjusted odds ratio (AOR) with 95\% confidence interval (CI).

Survival analysis, according to the patterns of CRP ratio response to antibiotic therapy and adequacy of antibiotic therapy, was performed using the Kaplan-Meier method. In order to compare survival distributions, the log-rank test was used. Significance was accepted at a p-value of $<0.05$.

\section{RESULTS}

During the study period, 47 out of 237 mechanically ventilated patients developed VAP with microbiological confirmation. Of the 47 patients, $13(27.7 \%)$ died in the ICU, and all deaths occurred while the patients were still on antibiotics and mechanically ventilated. The demographic characteristics of the VAP patients are shown in table 1 and the microorganisms isolated in table 2. In five cases, more than one pathogen was isolated. Patients were mechanically ventilated before VAP diagnosis for $11.7 \pm 8.5$ days and 13 were early-onset episodes [6].

Before the development of VAP, 29 out of 47 (61.7\%) patients had previously received antibiotics, 18 survivors and 11 nonsurvivors $(\mathrm{p}=0.046)$, for periods of $14.1 \pm 9.8$ and $13 \pm 6.5$ days, respectively $(p=0.928)$. VAP patients who had previously received antibiotics exhibited a mortality rate of $38 \%$, whereas, amongst those without previous antibiotics, it was $11 \%(\mathrm{p}=0.046)$.

At D0, the CRP, body temperature and WCC of survivors and nonsurvivors were not significantly different $(20.6 \pm 8.3$ versus $23.1 \pm 7.9 \mathrm{mg} \cdot \mathrm{dL}^{-1} \quad(\mathrm{p}=0.342), \quad 38.3 \pm 0.9$ versus $38.4 \pm 1.1^{\circ} \mathrm{C}$ $(p=0.807)$ and $13.7 \pm 6.1 \times 10^{3}$ versus $13.4 \pm 6.1 \times 10^{3}$ cells $\cdot \mu \mathrm{L}^{-1}$ $(p=0.967)$, respectively). Time-dependent analysis of CRP level and CRP ratio (fig. 2), from D0 to D7 of antibiotic therapy, showed steady and significant decreases in survivors, whereas, 


\begin{tabular}{|c|c|c|c|}
\hline \multirow[t]{2}{*}{ TABLE 1} & \multirow[b]{2}{*}{ Survivors } & \multirow[b]{2}{*}{ Nonsurvivors } & \multirow[b]{2}{*}{ p-value } \\
\hline & & & \\
\hline Subjects $n$ & 34 & 13 & \\
\hline Age yrs & $60.1 \pm 13.5$ & $66.5 \pm 12.6$ & 0.146 \\
\hline Males/females $n$ & $20 / 14$ & $11 / 2$ & 0.321 \\
\hline APACHE II score & $19.7 \pm 5.4$ & $22.5 \pm 6.4$ & 0.135 \\
\hline $\mathrm{Pa}, \mathrm{O}_{2} / \mathrm{Fl}_{1} \mathrm{O}_{2} \mathrm{mmHg}$ & $211 \pm 77$ & $142 \pm 46$ & 0.001 \\
\hline Reason for MV n & & & 0.41 \\
\hline Post-operative respiratory failure & 5 & 3 & \\
\hline AECB & 6 & 2 & \\
\hline Sepsis & 3 & 0 & \\
\hline Neurological disease or NMD & 1 & 1 & \\
\hline CAP\# & 3 & 3 & \\
\hline Coma & 1 & 0 & \\
\hline Cardiac failure & 5 & 0 & \\
\hline Trauma & 7 & 2 & \\
\hline Other" & 3 & 2 & \\
\hline Prior infection $n$ & 18 & 11 & 0.046 \\
\hline CRP antibiotic response $n$ & & & $<0.001$ \\
\hline Fast response & 10 & 0 & \\
\hline Slow response & 20 & 0 & \\
\hline Nonresponse & 2 & 7 & \\
\hline Biphasic response & 2 & 6 & \\
\hline
\end{tabular}

Data are presented as $n$ and mean $\pm \mathrm{SD}$, unless otherwise stated. APACHE: Acute Physiology, Age, and Chronic Health Evaluation; $\mathrm{Pa}_{2} \mathrm{O}_{2}$ : arterial oxygen tension; $\mathrm{Fl}_{1} \mathrm{O}_{2}$ : inspiratory oxygen fraction; $\mathrm{MV}$ : mechanical ventilation; $\mathrm{AECB}$ : acute exacerbation of chronic bronchitis; NMD: neuromuscular disease; CAP: community-acquired pneumonia; CRP: C-reactive protein. \#: isolates of Pseudomonas aeruginosa $(n=3)$, Klebsiella spp. $(n=1)$, methicillin-resistant Staphylococcus aureus $(n=1)$ and Escherichia coli $(n=1) ; "$ : cardiopulmonary resuscitation $(n=1)$, shock $(n=2)$ and poisoning $(n=2) .1 \mathrm{mmHg}=0.133 \mathrm{kPa}$.

in nonsurvivors, these parameters remained almost unchanged $(p<0.001$ and $p=0.001$, respectively). Over the same time period, body temperature likewise decreased in both groups $(p=0.799)$, although the body temperature of nonsurvivors was, on average, slightly lower than that of survivors. Analysis of WCC showed a significant difference between survivors and nonsurvivors $(p=0.001)$, but this finding was strongly influenced by three nonsurvivors with leukaemoid reactions. Excluding these patients from the WCC analysis, no significant difference was found between survivors and nonsurvivors $(\mathrm{p}=0.413)$.

When the CRP ratio of survivors and nonsurvivors was compared at different time points, significant differences were found at D4 $(\mathrm{p}=0.015), \mathrm{D} 5(\mathrm{p}=0.002), \mathrm{D} 6(\mathrm{p}=0.002)$ and D7 $(p=0.002$; fig. 2$)$. At $D 4$, the CRP ratio in survivors was 0.62 relative to the initial level $(\mathrm{p}<0.001)$, whereas, in nonsurvivors, it remained almost unchanged, at $0.98(\mathrm{p}=0.441)$. The AUC for the CRP ratio by D4 was 0.78 (95\% CI 0.62-0.91), whereas the AUCs of WCC and body temperature by D4 were 0.52 (95\% CI $0.34-0.71$ ) and 0.48 (95\% CI 0.28-0.69), respectively. The AUC of the CRP ratio by $\mathrm{D} 4$ was significantly greater than that of WCC and body temperature $(p=0.037$ and $p=0.031$, respectively). A CRP ratio by $\mathrm{D} 4$ of $>0.6$ was a marker of poor outcome, with a sensitivity of 0.92 , a specificity of 0.59 , a negative predictive value of 0.95 and a positive predictive value of 0.46 (positive likelihood ratio 2.24; negative likelihood ratio 0.13 ). In the multivariable analysis, only three variables, D4 CRP ratio (0.1 increment; AOR 1.401; 95\% CI 1.004-1.957; $\mathrm{p}=0.048)$, age (1-yr increment; AOR 1.135; 95\% CI 1.013-1.271; $\mathrm{p}=0.029)$ and D0 SOFA score (1-point increment; AOR 1.469; 95\% CI 1.014-2.127; $\mathrm{p}=0.042)$, were independent predictors of death (model $\mathrm{n}=47$; $\mathrm{AUC}=0.91$; goodness of fit $=0.932$ ). By D4, both body temperature and WCC were not significantly associated with outcome and were excluded from the model.

At the end of antibiotic therapy, the CRP concentration of survivors was $3.5 \pm 2.6 \mathrm{mg} \cdot \mathrm{dL}^{-1}$, whereas, in nonsurvivors, on the day of death, it was $26.2 \pm 8.2 \mathrm{mg} \cdot \mathrm{dL}^{-1}$ ( $\mathrm{p}<0.001$; fig. 3 ). Body temperature at the end of antibiotic therapy in survivors was similar to that in nonsurvivors on the day of death, $37.1 \pm 0.9$ and $37.3 \pm 0.9^{\circ} \mathrm{C}(p=0.207)$. Both survivors and nonsurvivors showed a significant decrease in body temperature $(p<0.001$ and $p=0.02$, respectively). Finally, the WCC of survivors at the end of antibiotic therapy was $9.8 \pm 3.2 \times 10^{3}$ cells $\cdot \mu \mathrm{L}^{-1}$, whereas, in nonsurvivors, on the day of death, it was $20.8 \pm 16.5 \times 10^{3}$ cells $\cdot \mu \mathrm{L}^{-1}(\mathrm{p}=0.034)$.

\section{CRP patterns of response to antibiotic therapy}

VAP patients were retrospectively divided according to four patterns of CRP ratio progression during antibiotic therapy (fig. 1). Ten patients were classified as showing a fast response, 20 a slow response, nine nonresponse and eight a biphasic response. Time-dependent analysis of the CRP ratio of the four different patterns showed that these patterns of progression were significantly different $(p<0.001)$. By D4, the CRP ratio was $0.23 \pm 0.12,0.79 \pm 0.33,1.03 \pm 0.19$ and $0.88 \pm 0.62$ in patients exhibiting a fast response, a slow response, nonresponse and a biphasic response pattern, respectively $(p<0.001)$. Conversely, during the same time period, no significant difference between the different patterns was found in the progression of WCC and body temperature $(p=0.613$ and $\mathrm{p}=0.369$, respectively).

All patients with a CRP ratio pattern of fast and slow response survived, whereas those with a pattern of nonresponse and biphasic response exhibited overall mortality rates of 78 and $75 \%$, respectively $(p<0.001)$. Finally, there was no association between the reason for mechanical ventilation or the presence of previous infection and the pattern of CRP ratio response to therapy $(p=0.154$ and $p=0.431$, respectively). At D0, acute respiratory distress syndrome (ARDS) criteria [23] were met by $15(44 \%)$ survivors and $10(77 \%)$ nonsurvivors $(p=0.044)$. The frequency of ARDS according to the four patterns of CRP ratio response to antibiotics, fast response, slow response, nonresponse and biphasic response, was 60, 45, 67 and 50\%, respectively $(\mathrm{p}=0.702)$.

\section{Adequacy of antibiotic therapy}

Furthermore, the effect of the adequacy of initial antibiotic therapy on outcome, as well as on CRP changes over time, was assessed. The overall mortality rate was $18.4 \%$ in the 38 patients initially treated with adequate antibiotics and $66.7 \%$ in the nine patients with initially inadequate antibiotic therapy $(p=0.025)$. The progression of CRP ratio according to 


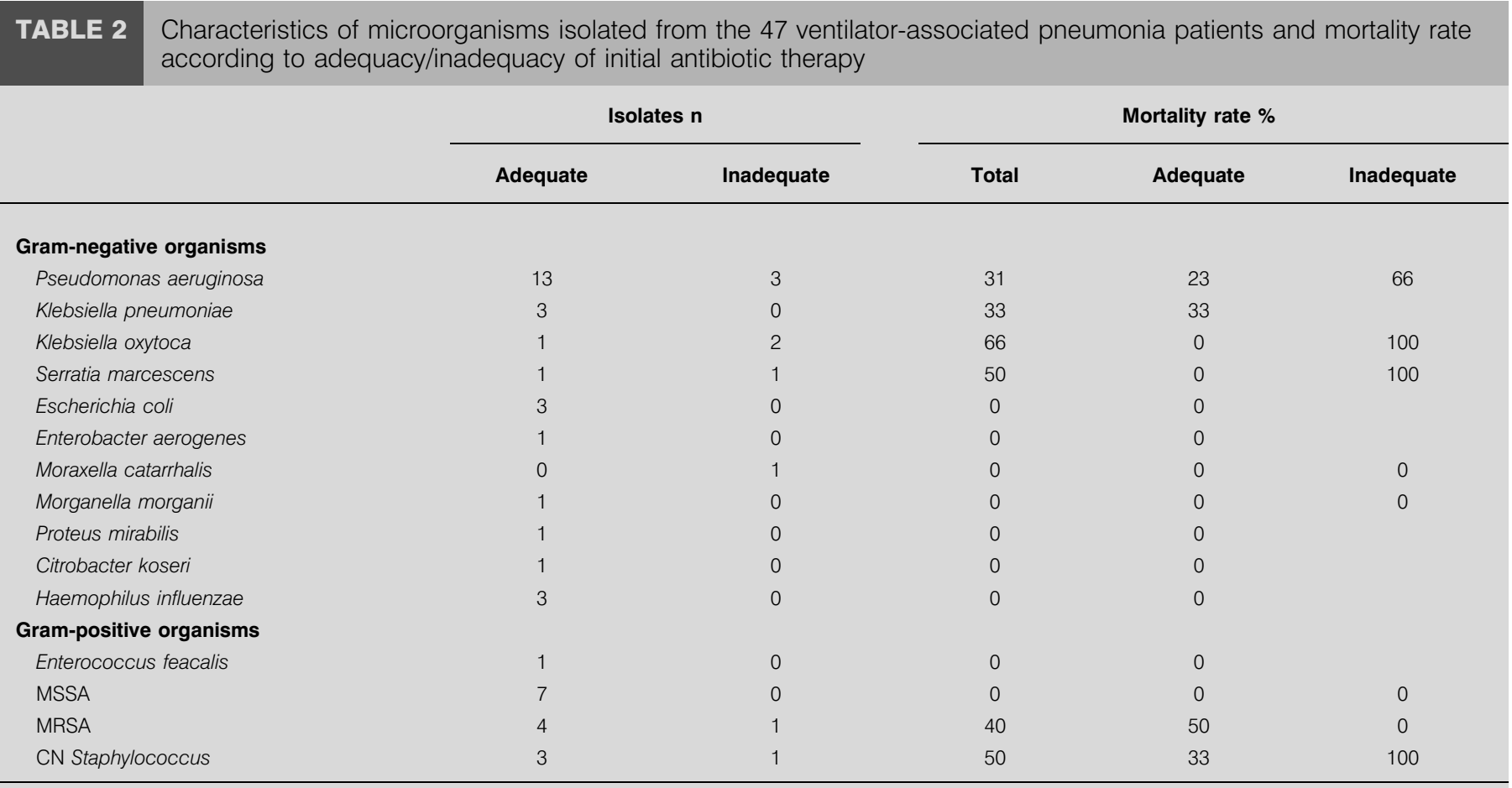

MSSA: methicillin-sensitive Staphylococcus aureus; MRSA: methicillin-resistant Staphylococcus aureus; CN: coagulase negative.

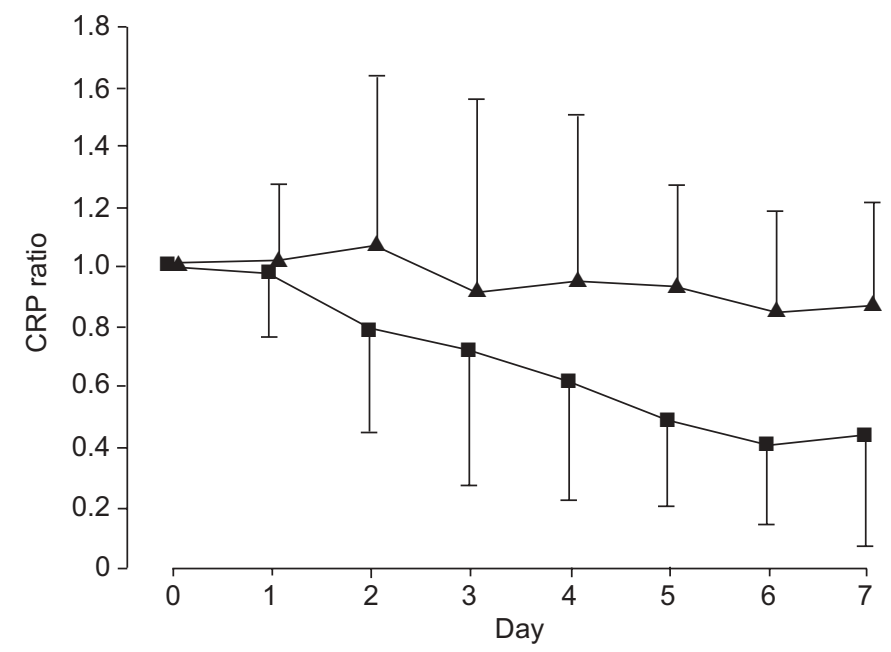

FIGURE 2. Time-dependent analysis of C-reactive protein (CRP) ratio during antibiotic therapy. Data are presented as mean \pm SD. From day 0 (D0) to D7 of antibiotic therapy, the CRP ratio was significantly different between survivors

and nonsurvivors $(\mathbf{\Lambda} ; p=0.001)$. At $\mathrm{D} 4$, the CRP ratio of survivors was 0.62 , relative to the initial value $(p<0.001)$. Calculation of contrast coefficients at different time points showed significant differences at D4 $(p=0.015), D 5(p=0.002), D 6$ $(p=0.002)$ and $D 7(p=0.002)$.

the adequacy of antibiotic therapy was compared from D0 to D7. Patients who initially received adequate antibiotics showed a marked CRP ratio decrease in comparison to those with initially inadequate therapy $(p<0.001)$. At D4, the CRP ratio of patients with adequate antibiotics was 0.6 ,

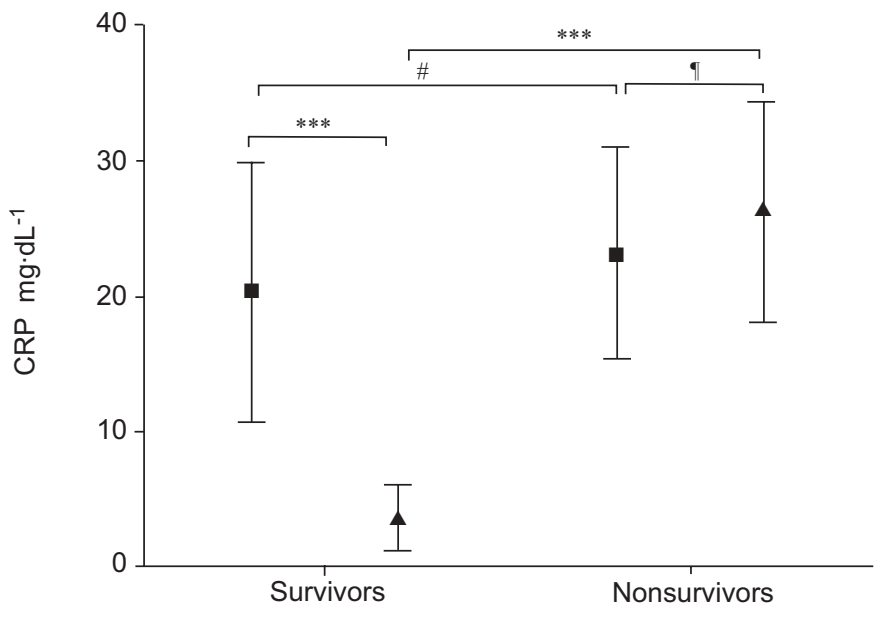

FIGURE 3. C-reactive protein (CRP) levels on the day of antibiotic prescription ( $\mathbf{\square}$; day 0) and last day of antibiotic therapy or death $(\mathbf{A})$ in survivors and nonsurvivors. Data are presented as mean \pm SD. ${ }^{*}: p=0.342 ; ~ \bullet: p=0.133$; *** $p<0.001$

whereas it remained well above 1.0 in patients on inadequate therapy.

The influence of the adequacy of antibiotic therapy on CRP ratio pattern of response was also analysed. All 10 patients with a pattern of fast response received adequate therapy, as well as $85 \%$ of the patients with a slow response. Conversely, $44 \%$ of the patients showing a nonresponse pattern and $25 \%$ with a pattern of biphasic response received inadequate antibiotic therapy $(\mathrm{p}=0.09)$. 


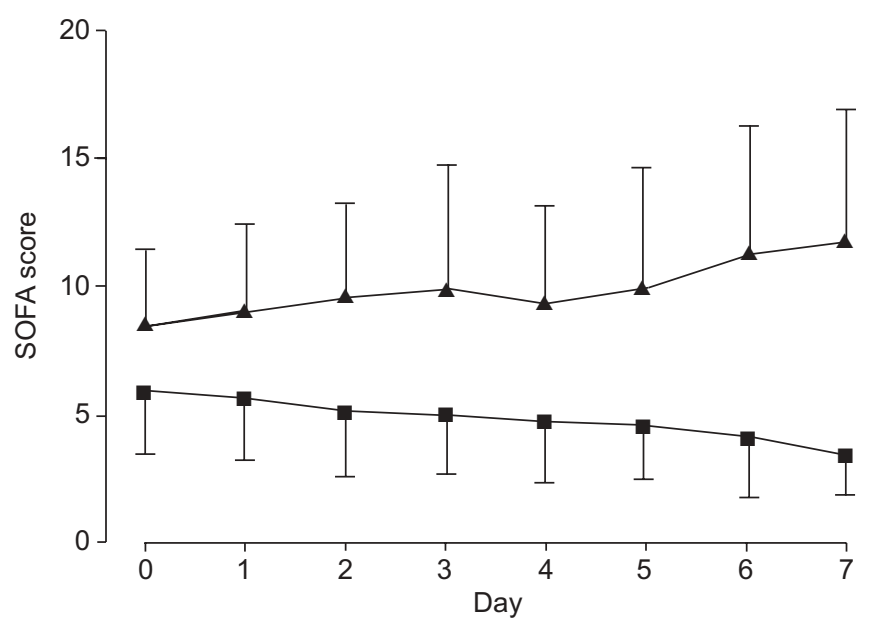

FIGURE 4. Sequential Organ Failure Assessment (SOFA) score between days 0 and 7 in survivors $(\boldsymbol{\square})$ and nonsurvivors $(\mathbf{\Lambda})$. Data are presented as mean \pm SD. $p<0.001$

\section{Correlation between outcome and CRP ratio patterns of response with clinical course}

Clinical progression during antibiotic therapy was monitored with daily measurements of SOFA score and $\mathrm{Pa}_{1} \mathrm{O}_{2} / \mathrm{FI}_{1} \mathrm{O}_{2}$. The result of time-dependent analysis of $\mathrm{Pa}_{1} \mathrm{O}_{2} / \mathrm{FI}_{1} \mathrm{O}_{2}$ from D0 to D7 of antibiotic therapy in survivors and nonsurvivors was not significantly different $(p=0.27)$. Conversely, during the same period, SOFA score progression was significantly different $(\mathrm{p}<0.001)$. In survivors, the SOFA score decreased steadily from $6 \pm 2.6$ at D0 to $3.4 \pm 1.5$ at D7 $(\mathrm{p}<0.001)$, whereas, in nonsurvivors, it increased progressively from $8.4 \pm 2.9$ to $11.6 \pm 5.6$ ( $p=0.1$; fig. 4 ).

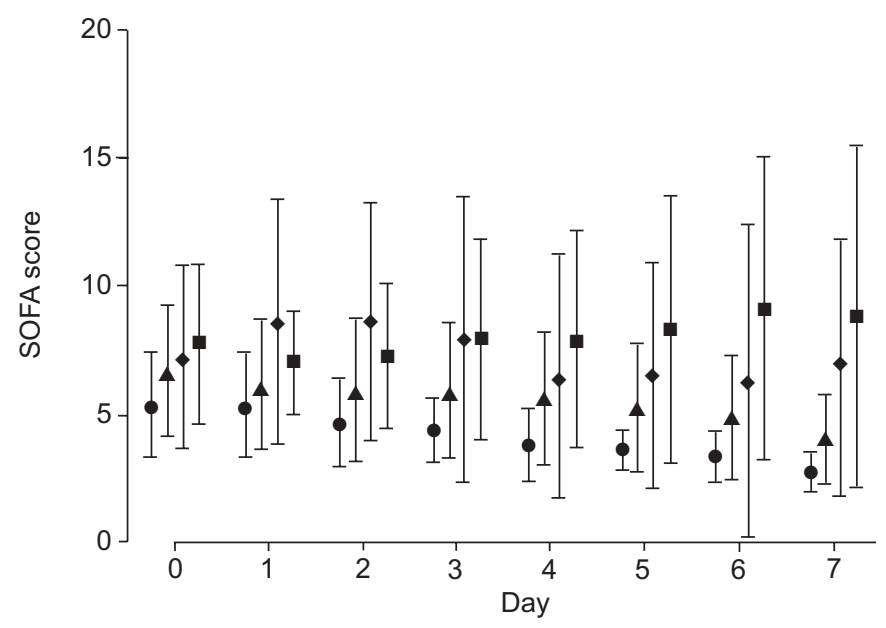

FIGURE 5. Sequential Organ Failure Assessment (SOFA) score between days 0 and 7 according to pattern of $\mathrm{C}$-reactive protein ratio response to antibiotics ( fast response; $\mathbf{\Lambda}$ : slow response; : nonresponse; $\mathbf{\square}$ : biphasic response). Data are presented as mean $\pm \mathrm{SD}$. These four patterns of progression were significantly different from each other $(p<0.001)$.
The SOFA score 3 days before VAP diagnosis was $5.7 \pm 2.5$ in survivors and $6.6 \pm 2.5$ in nonsurvivors $(p=0.369)$, and, in addition, SOFA score changes between D3 and D0 were not significant in survivors and nonsurvivors $(p=0.824$ and $\mathrm{p}=0.111$, respectively).

The time-dependent analysis of $\mathrm{Pa}_{2} \mathrm{O}_{2} / \mathrm{FI}_{1} \mathrm{O}_{2}$ for the four different CRP ratio patterns of response from D0 to D7 showed no significant differences between the different patterns $(p=0.94)$. However, the time-dependent analysis of SOFA score for the four patterns (fig. 5) showed that these patterns of progression were significantly different $(\mathrm{p}<0.001)$. The SOFA scores of patients with the fast and slow CRP ratio patterns decreased from $5.3 \pm 2.1$ and $6.7 \pm 2.6$ at D0 to $2.8 \pm 0.7$ and $4.0 \pm 1.6$ at $D 7$, respectively $(p=0.029$ and $p=0.001)$. Conversely, the SOFA scores of the patients showing nonresponse and biphasic CRP ratio patterns remained almost unchanged, or increased even further, from $7.1 \pm 3.7$ and $7.8 \pm 3.1$ at D0 to $7.0 \pm 4.6$ and $9.2 \pm 7.3$ at D7, respectively $(\mathrm{p}=0.715$ and $\mathrm{p}=0.91)$.

For each of the six organ systems included in the SOFA score, organ failure is defined as a score of $\geqslant 3$ [17]. Patients with VAP had at least one organ failure at D0 since all were mechanically ventilated, i.e. had a respiratory system score of $\geqslant 3$. Patients with the fast response pattern exhibited less severe VAP, as only three (30\%) showed additional organ failure. In contrast, in patients with the slow response pattern, $14(70 \%)$ presented another organ failure $(p=0.037)$. However, between D0 and D7, the SOFA score of patients with fast and slow response declined at a similar rate $(p=0.945)$. At D7, organ failures were present in only three $(30 \%)$ patients with the fast response pattern, whereas $13(65 \%)$ with the slow response pattern had at least one $(p=0.048)$. The duration of mechanical ventilation after antibiotic prescription was also analysed. Patients with a fast response were ventilated for $4.6 \pm 1.3$ days, whereas those with a slow response remained ventilated for $7.8 \pm 5.5$ days $(p=0.053)$.

\section{DISCUSSION}

In the present study, the clinical resolution of VAP, assessed by serial measurements of CRP, body temperature and WCC, after institution of antibiotic therapy, was evaluated in order to identify, early in the clinical course, patients with good and bad outcome. Currently, the evaluation of VAP resolution relies on essentially the same criteria as used in the diagnosis [24]. However, some of these criteria, such as body temperature, WCC and $\mathrm{Pa}_{1} \mathrm{O}_{2} / \mathrm{FI}_{1} \mathrm{O}_{2}$, are influenced by a number of noninfectious factors [25]. Based on the present findings, daily CRP measurements could be used as a marker of VAP resolution, and might be of some help in the clinical decisionmaking process for the reassessment of patients that fail to improve.

PUGIN et al. [26] proposed the CPIS, which relied on a combination of clinical signs plus tracheal aspirate Gram stain and culture, for VAP diagnosis. The cut-off and weight of each variable included in the score were set empirically, and a CPIS of $>6$ had a sensitivity of 0.93 for detecting VAP. However, the diagnostic accuracy of the CPIS has been successively challenged $[27,28]$. 
In a multicentre study, LuNA et al. [8] studied the performance of serial CPIS measurements during VAP resolution. Timedependent analysis showed that the CPIS of survivors was significantly lower at days 3 and 5 in comparison with nonsurvivors, but at day 7, no significant difference was found. Individual analysis of the CPIS components showed that only $\mathrm{Pa}, \mathrm{O}_{2} / \mathrm{FI}_{1} \mathrm{O}_{2}$ progression was significantly different at days 3 and 5 , but not at the end of the first week of therapy.

The value of CRP level changes over time has not yet been systematically investigated in critically ill patients. However, in several papers, the authors systematically recognised that decreases in CRP levels precede clinical improvement, whereas, conversely, failure of CRP levels to fall suggests infectious complication or ineffective/inappropriate treatment $[12,13,29-31]$. To the present authors' knowledge, there are only two studies evaluating the role of serial CRP determinations in monitoring response to therapy in critically ill patients. The first showed that a decrease in CRP levels by $\geqslant 0.25$ times from the previous day's level was a good marker of sepsis resolution [14]. The other found that a CRP concentration decrease of $\geqslant 5 \mathrm{mg} \cdot \mathrm{dL}^{-1}$ between admission and day 4 of antibiotic therapy was associated with clinical recovery [32].

Serial measurements of CRP, body temperature and WCC were performed in VAP patients from the day of antibiotic prescription, D0, to the day of death or the end of antibiotic therapy, dividing patients into survivors and nonsurvivors. It is important to realise that daily CRP measurements were performed not to predict outcome but to describe clinical course. From D0 to D7, CRP ratio showed a significant and steady decrease in survivors, whereas, in nonsurvivors, it remained elevated. In survivors, by D4, CRP ratio had decreased by almost 0.4 . Comparisons of ROC curves showed that the prognostic performance of CRP ratio by D4 was significantly better than that of body temperature and WCC. These results could be explained, at least in part, by the influence of noninfectious factors on body temperature and WCC [33]. A CRP ratio by D4 of $>0.6$ was associated with the diagnosis of nonresolving VAP. Additionally, multivariable logistic regression analysis showed that, among the variables studied, only CRP ratio by D4 was significantly and independently associated with outcome. The finding that serial measurements of both body temperature and WCC were poor predictors of VAP resolution has already been shown by LUNA et al. [8].

The evaluation of changes in clinical variables, such as SOFA score and $\mathrm{Pa}_{2} \mathrm{O}_{2} / \mathrm{FI}_{1} \mathrm{O}_{2}$, over time may be helpful in the assessment of the effect of different therapeutic interventions [31]. In the present study, it was not possible to reproduce the findings of LUNA et al. [8], as the time-dependent analysis of $\mathrm{Pa}_{\mathrm{a}} \mathrm{O}_{2} / \mathrm{FI}, \mathrm{O}_{2}$ from D0 to D7 did not discriminate between survivors and nonsurvivors. Conversely, a significant and steady decrease in SOFA score from D0 to D7 was found in survivors, whereas, in nonsurvivors, a progressive increase was observed. In this context, the correlation of CRP ratio with organ failure suggests that a decrease in CRP concentration, indicating resolution of the inflammatory process, was associated with a better outcome, whereas persistently elevated or increasing CRP levels, denoting ongoing inflammation, carried a poor prognosis.
Correlation of the CRP ratio patterns of response to antibiotics and outcome was also performed. It was found that the patterns of CRP ratio response were closely correlated with outcome. All patients with fast and slow response patterns survived, whereas the combined mortality rate of the patients showing the nonresponse and biphasic response patterns was $76.5 \%$. The analysis was carried further in that the relation between CRP ratio patterns of response and the SOFA score was studied. Patients with a fast response pattern showed less severe VAP than those with a slow response, but the rate of organ failure improvement after antibiotic prescription was parallel. In contrast, patients showing nonresponse and biphasic response patterns exhibited more severe VAP, and their SOFA score remained unchanged or increased even further over time. Finally, it was shown that the individual pattern of CRP ratio response to antibiotics was not influenced by the reason for mechanical ventilation, the presence of ARDS at the time of VAP diagnosis or the presence of previous infection. Therefore, the individual pattern of CRP ratio response to antibiotics appeared to be a reflection of VAP clinical course independent of other possible confounders.

The impact of initially adequate antibiotic therapy on mortality has been demonstrated by several studies [8, 15, 16, 24]. In the present group of VAP patients, those with initially adequate antibiotic therapy exhibited a better outcome than did those with initially inadequate therapy. At D4, the CRP ratio of patients with adequate therapy showed a marked decrease, whereas, in patients with inadequate therapy, the CRP ratio remained elevated. The adequacy of the initial antibiotic therapy also had a marked influence on the CRP ratio patterns of response.

Some limitations of the present investigation should be noted. First, this was a cohort single-centre observational pilot study using variables collected daily at the bedside to evaluate VAP clinical course. The present study deals with a frequently asked question in the management of VAP patients after prescription of antibiotics, either improving or deteriorating, which, as yet, has not been fully addressed in the literature. As a result, the present results could not be compared with any other previously published study. Secondly, the distribution of the present VAP patients between early and late onset was somewhat unbalanced. Hence, some agents frequently isolated from early onset episodes, such as Streptococcus pneumoniae, were absent from among the microorganisms isolated from the present patients. Thirdly, a mixed group of medical and surgical VAP patients was examined. It is possible that other types of patient (e.g. solid organ transplant recipients, febrile neutropenics and those undergoing allogeneic stem cell transplantation), as well as infection (e.g. bloodstream infections and viral infections), may show different CRP concentration time courses. Finally, some possible CRP level confounders, such as acute myocardial infarction, were not systematically monitored. Although, myocardial necrosis and myocardial ischaemia are well-known stimuli for CRP production [34], its magnitude is not equivalent to that of the infection-mediated CRP response, in particular of bacterial origin $[12,34,35]$. Thus, the impact of unsuspected cardiovascular events in the present study would be negligible. 
From the present study, it is concluded that daily C-reactive protein concentration measurement after prescription of antibiotic therapy is useful in the identification, as early as day 4 , of ventilator-associated pneumonia patients with poor outcome, and performs better than the commonly used markers of infection, such as body temperature and white cell count. In addition, recognition of the pattern of C-reactive protein ratio response to therapy provides more information about individual clinical course, improving or worsening, as well as rate of improvement. Therefore, the present results suggest that, in ventilator-associated pneumonia patients with rapid C-reactive protein ratio decline, a shorter duration of antibiotic therapy could be equally effective, reducing the risks of emergence of resistant strains and costs. Conversely, in patients showing the patterns of nonresponse and biphasic response, an aggressive diagnostic and therapeutic approach should be attempted in order to prevent further clinical deterioration. If these findings are confirmed, the duration of antibiotic therapy could be individualised to each patient's clinical response.

\section{REFERENCES}

1 Vincent JL, Bihari DJ, Suter PM, et al. The prevalence of nosocomial infection in intensive care units in Europe. Results of the European Prevalence of Infection in Intensive Care (EPIC) Study. EPIC International Advisory Committee. JAMA 1995; 274: 639-644.

2 Cook D. Ventilator associated pneumonia: perspectives on the burden of illness. Intensive Care Med 2000; 26: Suppl. 1, S31-S37.

3 Fagon JY, Chastre J, Vuagnat A, Trouillet JL, Novara A, Gibert C. Nosocomial pneumonia and mortality among patients in intensive care units. JAMA 1996; 275: 866-869.

4 Ewig $S$, Bauer $T$, Torres A. The pulmonary physician in critical care - 4: nosocomial pneumonia. Thorax 2002; 57: 366-371.

5 Dennesen PJ, van der Ven AJ, Kessels AG, Ramsay G, Bonten MJ. Resolution of infectious parameters after antimicrobial therapy in patients with ventilator-associated pneumonia. Am J Respir Crit Care Med 2001; 163: 1371-1375.

6 Hospital-acquired pneumonia in adults: diagnosis, assessment of severity, initial antimicrobial therapy, and preventive strategies. A consensus statement, American Thoracic Society, November 1995. Am J Respir Crit Care Med 1996; 153: 1711-1725.

7 Montravers P, Fagon JY, Chastre J, et al. Follow-up protected specimen brushes to assess treatment in nosocomial pneumonia. Am Rev Respir Dis 1993; 147: 38-44.

8 Luna CM, Blanzaco D, Niederman MS, et al. Resolution of ventilator-associated pneumonia: prospective evaluation of the clinical pulmonary infection score as an early clinical predictor of outcome. Crit Care Med 2003; 31: 676-682.

9 Halm EA, Fine MJ, Marrie TJ, et al. Time to clinical stability in patients hospitalized with community-acquired pneumonia: implications for practice guidelines. JAMA 1998; 279: 1452-1457.

10 Vigushin DM, Pepys MB, Hawkins PN. Metabolic and scintigraphic studies of radioiodinated human C-reactive protein in health and disease. J Clin Invest 1993; 91: 1351-1357.
11 Povoa P, Coelho L, Almeida E, et al. C-reactive protein as a marker of infection in critically ill patients. Clin Microbiol Infect 2005; 11: 101-108.

12 Povoa P. C-reactive protein: a valuable marker of sepsis. Intensive Care Med 2002; 28: 235-243.

13 Cox ML, Rudd AG, Gallimore R, Hodkinson HM, Pepys MB. Real-time measurement of serum C-reactive protein in the management of infection in the elderly. Age Ageing 1986; 15: 257-266.

14 Yentis SM, Soni N, Sheldon J. C-reactive protein as an indicator of resolution of sepsis in the intensive care unit. Intensive Care Med 1995; 21: 602-605.

15 Kollef MH, Sherman G, Ward S, Fraser VJ. Inadequate antimicrobial treatment of infections: a risk factor for hospital mortality among critically ill patients. Chest 1999; 115: 462-474.

16 Ibrahim EH, Sherman G, Ward S, Fraser VJ, Kollef MH. The influence of inadequate antimicrobial treatment of bloodstream infections on patient outcomes in the ICU setting. Chest 2000; 118: 146-155.

17 Vincent JL, de Mendonca A, Cantraine F, et al. Use of the SOFA score to assess the incidence of organ dysfunction/ failure in intensive care units: results of a multicenter, prospective study. Working group on "sepsis-related problems" of the European Society of Intensive Care Medicine. Crit Care Med 1998; 26: 1793-1800.

18 Meduri GU, Chastre J. The standardization of bronchoscopic techniques for ventilator-associated pneumonia Chest 1992; 102: 557S-564S.

19 Chastre J, Fagon JY, Bornet-Lecso M, et al. Evaluation of bronchoscopic techniques for the diagnosis of nosocomial pneumonia. Am J Respir Crit Care Med 1995; 152: 231-240.

20 Hanley JA, McNeil BJ. The meaning and use of the area under a receiver operating characteristic (ROC) curve. Radiology 1982; 143: 29-36.

21 Swets JA. Measuring the accuracy of diagnostic systems. Science 1988; 240: 1285-1293.

22 Hanley JA, McNeil BJ. A method of comparing the areas under receiver operating characteristic curves derived from the same cases. Radiology 1983; 148: 839-843.

23 Bernard GR, Artigas A, Brigham KL, et al. The AmericanEuropean Consensus Conference on ARDS. Definitions, mechanisms, relevant outcomes, and clinical trial coordination. Am J Respir Crit Care Med 1994; 149: 818-824.

24 Ferrer M, Ioanas M, Torres A. Evaluation of non-responding patients with ventilator-associated pneumonia. In: Vincent JL, ed. Yearbook of Intensive Care and Emergency Medicine. Berlin, Springer, 2002; pp. 365-375.

25 Garrard CS, A'Court CD. The diagnosis of pneumonia in the critically ill. Chest 1995; 108: 17S-25S.

26 Pugin J, Auckenthaler R, Mili N, Janssens JP, Lew PD, Suter PM. Diagnosis of ventilator-associated pneumonia by bacteriologic analysis of bronchoscopic and nonbronchoscopic "blind" bronchoalveolar lavage fluid. Am Rev Respir Dis 1991; 143: 1121-1129.

27 Fabregas N, Ewig S, Torres A, et al. Clinical diagnosis of ventilator associated pneumonia revisited: comparative validation using immediate post-mortem lung biopsies. Thorax 1999; 54: 867-873.

28 Fartoukh M, Maitre B, Honore S, Cerf C, Zahar JR, BrunBuisson C. Diagnosing pneumonia during mechanical 
ventilation: the clinical pulmonary infection score revisited. Am J Respir Crit Care Med 2003; 168: 173-179.

29 Hogarth MB, Gallimore R, Savage $\mathrm{P}$, et al. Acute phase proteins, $\mathrm{C}$-reactive protein and serum amyloid A protein, as prognostic markers in the elderly inpatient. Age Ageing 1997; 26: 153-158.

30 Ugarte H, Silva E, Mercan D, De Mendonca A, Vincent JL. Procalcitonin used as a marker of infection in the intensive care unit. Crit Care Med 1999; 27: 498-504.

31 Lobo SM, Lobo FR, Bota DP, et al. C-reactive protein levels correlate with mortality and organ failure in critically ill patients. Chest 2003; 123: 2043-2049.
32 Reny JL, Vuagnat A, Ract C, Benoit MO, Safar M, Fagon JY. Diagnosis and follow-up of infections in intensive care patients: value of C-reactive protein compared with other clinical and biological variables. Crit Care Med 2002; 30: 529-535.

33 Abramson N, Melton B. Leukocytosis: basics of clinical assessment. Am Fam Physician 2000; 62: 2053-2060.

34 Pepys MB, Hirschfield GM. C-reactive protein: a critical update. J Clin Invest 2003; 111: 1805-1812.

35 Pepys MB, Baltz ML. Acute phase proteins with special reference to $\mathrm{C}$-reactive protein and related proteins (pentaxins) and serum amyloid A protein. Adv Immunol 1983; 34: 141-212. 